\title{
Infecções por micobactérias não tuberculosas no Hospital Nereu Ramos, centro de referência em Santa Catarina
}

\author{
Camilo Fernandes ${ }^{\prime}$, Carlos Rodrigo Zárate-Bladés ${ }^{2}$
}

1- Estudante de Mestrado do Programa de Pós-Graduação em Ciências Médicas, Centro de Ciências da Saúde, Universidade Federal de Santa Catarina (UFSC), Campus Reitor João David Ferreira Lima, Florianópolis, Brasil.

2- Professor do Departamento de Microbiologia, Imunologia e Parasitologia do Centro de Ciências Biológicas, membro permanente do Programa de Pós-Graduação em Biotecnologia e Biociências do Centro de Ciências Biológicas e do Programa de Pós-Graduação em Ciências Médicas, Centro de Ciências da Saúde, Universidade Federal de Santa Catarina (UFSC), Campus Reitor João David Ferreira Lima, Florianópolis, Brasil.

\begin{abstract}
RESUMO
Esta série especial de ensaios apresenta textos produzidos pelos alunos do Programa de Pós-Graduacão em Ciências Médicas na disciplina "Seminários de Pesquisa". Solicitou-se aos alunos que apresentassem, em primeira pessoa, sua motivação e trajetória que os conduziram ao trabalho na pós-graduação, bem como a pergunta e a metodologia de seus projetos de pesquisa, com o principal objetivo de inspirar os estudantes de graduação à carreira de pesquisadores. Neste ensaio, o mestrando apresenta seu interesse pelas infecções causadas por micobatérias não-tuberculosas em sua especialidade médica e como pretende pesquisar sobre suas características em nossa população.
\end{abstract}

DOI: https://doi.org/10.32963/bcmufsc.v5i6.3846

Indexadores: Ensaio; Educação de Pós-Graduação em Medicina; Micobactérias não Tuberculosas Submetido em 22/11/19; aceito para publicação em 4/12/19 Autor para correspondência: Camilo Fernandes. E-mail: camilofer28@yahoo.com.br

\section{Formei-me no curso de medicina da Universidade} Federal de Santa Catarina (UFSC) em agosto de 2000, e logo após minha formatura iniciei minhas atividades profissionais trabalhando como clínico geral em cidades pequenas no Sul de Santa Catarina. Após três anos da formatura, família formada e alguma experiência de médico do interior, retornei à grande Florianópolis para aperfeiçoar a formação médica realizando a tão desejada "residência". Fiz a especialização em medicina interna no Hospital Regional de São José, e na sequência a residência em pneumologia no Hospital Universitário (HU) da UFSC, quando já era médico plantonista no Hospital Nereu Ramos (HNR), hospital da Secretaria de Estado da Saúde de Santa Catarina, referência em doenças infecciosas e pulmonares, onde trabalho até os dias atuais.

Assim que terminei a residência em pneumologia fui convidado a ministrar aulas para o curso de medicina nesta especialidade na Universidade do Sul do Estado de Santa Catarina (UNISUL), na Palhoça (campus Pedra Branca) e em Tubarão. Já são 11 anos como professor universitário.
Trabalhando como pneumologista tive a experiência de tratar doenças raras e de difícil manejo, casos de Tuberculose (TB) com resistências diversas, entre elas pacientes com TB Multidroga Resistente (TB-MDR), TB Extensivamente Resistente (TB XDR) e casos de micobacterioses não tuberculosas. Essas doenças acometem geralmente pacientes com agravos pulmonares ou imunossuprimidos e observamos um aparente aumento da incidência dos casos de micobacterioses que não são TB.

O termo "micobactéria não tuberculosa" (MNT) refere-se a membros do gênero Mycobacterium que não fazem parte do complexo M. tuberculosis e M. leprae ${ }^{1}$. É cada vez mais frequente na literatura médica o relato de isolamento de MNT em espécimes clínicos, quando solicitada a pesquisa de bacilo ácido álcool resistente (BAAR) e cultura do material estudado, durante a investigação de um quadro clínico com suspeita de TB. Tais organismos são tipicamente ambientais e pouco patogênicos para humanos, mas podem ser responsáveis por doenças oportunistas em indivíduos que apresentam condições predisponentes ${ }^{2}$. As MNT são responsáveis por infecções pulmonares na sua maioria, 
embora a relevância das micobacterioses envolvendo outras partes do corpo esteja aumentando. 0 risco de infecções disseminadas que caracterizam pacientes imunocomprometidos é bem conhecido, mas também podem acontecer com acometimento mais localizado, como por exemplo em linfonodos, pele e tecidos subcutâneos, bem como ossos e articulações, que também são alvos importantes da infecção por MNT. As infecções por MNT são um importante causa de morbidade e mortalidade, muitas vezes sob a forma de doença pulmonar progressiva ${ }^{3}$. Vários estudos realizados em diferentes partes do mundo indicam que as infecções por MNT são um problema emergente de saúde global. No Brasil os dados são escassos, mas apontam na mesma direção. Em relação ao estado de SC, não existem dados epidemiológicos publicados sobre infecções por MNT. Além disso, os poucos dados do nosso país apresentam diferentes prevalências regionais de espécies de MNT envolvidas em infecções ${ }^{4}$. Sendo o Brasil um país de dimensões continentais, as diferenças demográficas devem ser consideradas junto com a compreensão da história natural desses pacientes em um contexto regional.

As infecções por MNT e muitos aspectos desta doença são difíceis de entender, desafiando explicações fáceis e requerendo informações detalhadas para construir um contexto adequado para sua interpretação e compreensão. Muitos aspectos da doença por MNT desafiam o tipo de análise baseada em evidências e, consequentemente, também se torna muito desafiador obter conclusões e recomendações a serem feitas nesses casos. 0 necessário acúmulo de informações para atingir esse objetivo (obtenção de conclusões baseados em diretrizes rigorosas nas MNT) simplesmente ainda não está disponível ${ }^{5}$. Os médicos que enfrentam difíceis decisões de gestão de MNT ainda precisam de auxílio e até mesmo a orientação imperfeita da "opinião de especialistas", e este é o objetivo de nosso trabalho, não se abstendo de opinar, informar, analisar e reanalisar o que assistimos diariamente no ambulatório de micobacteriose atípica que conduzimos há mais de 10 anos em Santa Catarina (SC).

Ser professor universitário, trabalhar em um centro de referência em doenças pulmonares, e vivenciar o desafio que representa o atendimento dos casos de MNT, foram os estímulos para procurar o aperfeiçoamento da minha formação como médico, buscando a Pós-Graduação em Ciências Médicas. Conto com a orientação do professor Carlos Rodrigo Zárate-Bladés. Junto com o meu orientador e com colegas que me acompanharam durante o trabalho do HNR, temos conseguido ampliar as metas do nosso estudo inicial e realizar parcerias de forma a estudar nossos pacientes também de forma prospectiva a partir de 2019. Nossos colaboradores incluem profissionais do LACEN (Darcita Buerger Rovaris, Sabrina Gonçalves, Luciane Cauduro Lima e Sandra Bianchini Fernandes), HU-UFSC (Profa Dr ${ }^{a}$ Maria Luiza Bazzo, Dra Mara Cristina Scheffer e mestrando Juliano dos Santos) e do próprio HRN (Dr. Cleyton Gregory da Silva e Enfa Zuleide Amaral Minella).

Dessa forma, o presente estudo (parecer aprovado CAAE 10950919.5.0000.0121 do Comitê de Ética em Pesquisa com Seres Humanos) será o primeiro a realizar uma análise detalhada considerando todos os casos atendidos no centro de referência dessas infecções desde a sua instalação, considerando aspectos epidemiológicos, clínicos e microbiológicos. Pretendemos que os dados gerados sejam disponibilizados para auxiliar no planejamento de melhorias no diagnóstico, tratamento e prevenção de infecção por MNT, especialmente para o médico na "linha de frente", assim como para melhorar o entendimento da epidemiologia e circulação desses microrganismos no nosso estado, e através disso, contribuir também com os gestores nacionais e regionais de saúde.

\section{Referências}

1. Wallace Jr RJ, O'Brien R, Glassroth J, Raleigh J, Dutt A. Diagnosis and treatment of disease caused by nontuberculous mycobacteria. Am J Respir Crit Care Med 1990; 142: 940-53.

2. Tortoli, E. Clinical manifestations of nontuberculous mycobacteria infections. Clin Microbiol Infec 2009; 15: 906-10.

3. Cowman S, Van Ingen J, Griffith DE, Loebinger MR. Nontuberculous mycobacterial pulmonary disease. Eur Respir J 2019; $54:$ 1-23.

4. De Mello KGC, Mello FCQ, Borga L et al. Clinical and therapeutic features of pulmonary nontuberculous mycobacterial disease, Brazil, 19932011. Emerg Infect Dis 2013; 19: 393-9.

5. Griffith DE. Nontuberculous Mycobacterial Disease. A comprehensive approach to diagnosis and management. Cham, Switzerland: Springer Nature, 2019. E-book. Disponível em: https://link.springer.com/book/10.1007\%2F978-3-319-93473-0. 


\section{Sobre o "Boletim do Curso de Medicina da UFSC"}

\section{Submissões para publicação:}

Preferencialmente online através do sistema Open Journals System. Acesso em: http://ojs.sites.ufsc.br/index.php/medicina (crie seu login e senha para acesso à revista através da página de cadastro, clicando em "Sobre" e em "Submissões"), ou por e-mail direto ao editor, com os arquivos em anexo.

\section{Diretrizes para Autores}

Trabalhos enviados para publicação devem ser montados em arquivo doc ou .docx, em língua portuguesa, com a seguinte estrutura:

Página de rosto: indicando a sessão em que pretende publicar o trabalho (veja detalhes das sessões em "Políticas de Sessão"), título, identificação dos autores em ordem de citação (nome completo, setor de trabalho, titulação e email de contato), endereço para correspondência do autor responsável pelos contatos.

A declaração de conflitos de interesse dos autores deve aparecer na folha de rosto e será publicada junto ao trabalho. Incluir fontes de apoio financeiro ao trabalho e citar se os autores possuem vinculação a empresas/coletivos/partidos que tenham interesse na divulgação das informações do trabalho.

Deve ser anexado um documento .pdf contendo o seguinte texto: "Declaração de concordância - os autores abaixo assinados declaram que concordam com a publicação do trabalho intitulado (escrever o título do trabalho) em "Boletim do Curso de Medicina da UFSC" e que aceitam as normas de publicação neste periódico. Os autores declaram os seguintes conflitos de interesse: (declarar ligação a empresas/coletivos/partidos/movimentos com interesse na divulgação das informações do trabalho). Cidade, data - Nome completo, e-mail e assinatura de todos os autores"

Segunda página (esta, em português e em inglês): Resumo com até 250 palavras (estruturado da mesma forma que 0 artigo), seguido de três a seis palavras-chave, separadas por ponto-e-vírgula, para indexação (baseadas no DECs - descritores em ciências da saúde)

Em seguida, o artigo (com o máximo de 3000 palavras). Se artigo original, estruturado em seções (Introdução, Objetivos, Método, Resultados, Discussão e Conclusões). Artigos de revisão podem ter estrutura Introdução, Método, Revisão e Conclusões. As seções "artigos", "o que o residente lê" e "cartas" tem estrutura livre e não exigem resumo. Na seção "infográfico", aceita-se o trabalho na forma de ilustração, que deve conter todas as informações relevantes, incluindo nomes dos autores e referências.

Trabalhos envolvendo pesquisa com seres humanos ou animais devem mencionar a aprovação no respectivo Comitê de Ética em Pesquisa. Ensaios clínicos devem mencionar o registro em órgão competente.

O artigo deve ser escrito em fonte Tymes New Roman, tamanho 12, espaço simples. As referências devem seguir a norma de Vancouver, conforme divulgadas nas orientações para o Trabalho de Conclusão de Curso da Medicina (http://www.medicina.ufsc.br/files/2011/11/NORMAS-TCC-MEDUFSC-2011.pdf) e nas orientações de normatização de trabalhos acadêmicos da Biblioteca Universitária (http://portal.bu.ufsc.br/normalizacao/), sendo numeradas ao longo do texto em algarismos arábicos sobrescritos.

Figuras e tabelas devem seguir as mesmas normas do TCC da medicina (http://www.medicina.ufsc.br/files/2011/11/NORMAS-TCC-MEDUFSC-2011.pdf). Tabelas podem ser incorporadas no mesmo arquivo do texto; figuras podem ser incorporadas no texto, mas também devem ser encaminhadas como "arquivo suplementar". 